\title{
Insulinomatosis: una causa muy rara de tumor neuroendocrino pancreático
}

\author{
Insulinomatosis: a very rare cause of pancreatic neuroendocrine tumor
}

\author{
Jaramillo Chacón $H,{ }^{1}$ González Devia $D,{ }^{2}$ López Panqueva $R P,{ }^{3}$ Cañón Solano $D,{ }^{3}$ \\ Aguirre Matallana $D,{ }^{4}$ Rey Rubiano AM, ${ }^{5}$ Segovia Gómez $J M,{ }^{6}$ Dussan Flórez $R F^{7}$
}

${ }^{1} M D$, Pregrado de medicina, Universidad de los Andes. Bogotá D. C., Colombia.

${ }^{2} \mathrm{MD}, \mathrm{Ms} C$, medicina interna, endocrinología. Hospital Universitario Fundación Santa Fe de Bogotá, Universidad de los Andes.

${ }^{3} M D$, patología quirúrgica. Hospital Universitario Fundación Santa Fe de Bogotá, Universidad de los Andes.

${ }^{4} \mathrm{MD}$, radiología, Hospital Universitario Fundación Santa Fe de Bogotá.

${ }^{5} \mathrm{MD}$, medicina interna, gastroenterología. Hospital Universitario Fundación Santa Fe de Bogotá, Universidad de los Andes.

${ }^{6} \mathrm{MD}$, medicina interna, hematooncología. Hospital Universitario Fundación Santa Fe de Bogotá, Universidad de los Andes.

${ }^{7} M D$, radioterapia. Hospital Universitario Fundación Santa Fe de Bogotá, Universidad de los Andes.

Autor de correspondencia: Deyanira González Devia Correo electrónico: deyaniragonzalezdevia@yahoo.com Fecha de recepción: 2/05/2019

Fecha de aceptación: 2/02/2020

\section{Resumen}

Los tumores neuroendocrinos pancreáticos representan del 2-10\% de todos los tumores del páncreas y aproximadamente el $7 \%$ de todos los tumores neuroendocrinos. Estos se clasifican como funcionales o no funcionales según la presencia o ausencia de síndromes clínicos asociados con la hipersecreción hormonal. Los insulinomas son los tumores neuroendocrinos pancreáticos funcionales más frecuentes (45\% de los casos) y la causa más frecuente de hipoglucemia hiperinsulinémica endógena persistente en adultos. Además, el $10 \%$ de los tumores neuroendocrinos pancreáticos se asocian con neoplasia endocrina múltiple tipo 1. La insulinomatosis es una entidad clínica distinta en la que existen múltiples insulinomas.

Objetivos: exponer los casos reportados hasta el momento de insulinomatosis y describir las causas genéticas, las características clínicas, el tratamiento, y el pronóstico de la insulinomatosis.
Métodos: se realizó una búsqueda sobre insulinomatosis y los factores que controlan la proliferación de las células $\beta$ en las bases de datos PubMed, Medline y Google Scholar hasta Julio 2020.

Resultados: 108 casos con insulinomatosis se han reportado hasta la fecha, siendo recurrente y rara vez malignos. Múltiples protooncogenes y supresores de tumores controlan de forma local y sistémica el crecimiento de las células $\beta$; sin embargo, solo la mutación de MafA en p.Ser64Phe ha sido asociada.

Conclusión: la insulinomatosis se caracteriza por la aparición sincrónica y metacrónica de insulinomas. Tiene un fenotipo histológico, clínico y genético diferente a los tumores neuroendocrinos pancreáticos; la mutación MEN-1 es negativa; puede ser esporádica o hereditaria; y MafA podría ser una mutación conductora.

Palabras clave: hipoglucemia, insulinoma, nesidioblastosis, insulinomatosis, tumor neuroendocrino.

\section{Abstract}

Pancreatic neuroendocrine tumors represent 2-10\% of all tumors of the pancreas and approximately $7 \%$ of all neuroendocrine tumors. They are classified as functioning or non-functioning depending on the presence or absence of clinical syndromes associated with hormonal hypersecretion. Insulinomas are the most frequent functioning pancreatic neuroendocrine tumors ( $45 \%$ of cases), and the most frequent cause of persistent hyperinsulinemic hypoglycemia in adults. Furthermore, $10 \%$ of pancreatic neuroendocrine tumors are associated with multiple endocrine neoplasia type 1 (MEN-1). Insulinomatosis is a distinct clinical entity in which there are multiple insulinomas.

objectives: Present the cases reported to the date of insulinomatosis and describe the genetic causes, clinical characteristics, treatment and prognosis of insulinomatosis.

Methods: A search about insulinomatosis and the factors that govern beta cells proliferation was conducted in the 
databases of PubMed, Medline and Google Scholar until July 2020.

Results: 108 cases of patients with insulinomatosis have been reported to date, is recurrent and rarely malignant; multiple protooncogenes and tumor suppressors control locally and systemically the growth of beta cells; however, few have been studied in the tumoral landscape, and MafA mutation in p.Ser64Phe has been involved.

Conclusion: Insulinomatosis is characterized by the synchronous and metachronous occurrence of insulinomas; it has a histological, clinical and genetic phenotype different from other pancreatic neuroendocrine tumors. MEN-1 genetic testing is negative, can be sporadic or hereditary, and MafA could be a driver mutation.

Keywords: hypoglycemia, insulinoma, nesidioblastosis, insulinomatosis, pancreatic neuroendocrine.

\section{Introducción}

Desde hace 2 siglos (231 años) se ha estudiado la normalidad y anormalidad de las células $\beta$, incluyendo la hiperglucemia y la hipoglucemia asociada a neoplasias. Uno de los momentos más apasionantes de la historia de la medicina fue en 1922, cuando Banting y Best aislaron la insulina o isletina del extracto de páncreas de perro (1), y nueve meses después describieron los efectos adversos (hipoglucemia) de la dosis no estandarizada del extracto. Esta fue la primera vez que se pudo evidenciar los efectos de un exceso de insulina. Se observó nerviosismo, temblor, hambre, debilidad, sudoración, palidez/rubor, ansiedad, lipotimia, vértigo, diplopía y, en algunos casos, excitación, inestabilidad emocional, afasia sensorial y motora, disartria, delirio, desorientación, confusión, e inconsciencia; sin mencionar las convulsiones. Esta condición se aliviaba con la administración de carbohidratos y evitando el exceso de dosis (2).

En 1923, Harris sugirió la existencia del hiperinsulinismo endógeno y en 1924 publicó 4 casos de hipoglucemia cuyos síntomas mejoraron con la alimentación (3). Adicionalmente, Parker y Finley reportaron 10 casos de hipoglucemias y manifestaciones nerviosas graves tratadas con glucosa oral (4).

Posteriormente, en 1933 Wolf y colaboradores describieron el caso de un niño de 10 años con historia de convulsiones e hipoglucemias extirpándole 3 tumores de islotes (5) y entre 1932 y 1934 se reconoció la existencia de otras causas de hipoglucemias como alteraciones adrenales, pituitarias, tiroideas, hepáticas, depleciones de las reservas de glucógeno, sarcopenias y distrofias (6-11).

La hipoglucemia hiperinsulinémica endógena tumoral constituye un espectro que va desde la nesidioblastosis, el insulinoma solitario esporádico, los insulinomas múltiples heredofamiliares y la insulinomatosis esporádica y familiar (Figura 1). Estas patologías se pueden presentar metacrónicas o sincró- nicas; menos frecuente se presenta, la combinación de insulinoma y nesidioblastosis (12-14), y de prediabetes/diabetes mellitus tipo 2 (DM2) e insulinoma/insulinomatosis (15-18).

Figura 1. Espectro etiológico de la hipoglucemia hiperinsulinémica tumoral producida por las células $\beta$; donde el insulinoma solitario corresponde al $80 \%$, insulinoma maligno al $8 \%$, insulinomas múltiples en MEN-1 $5 \%$, insulinomatosis $5 \%$ y nesidioblastosis $2 \%$. Las causas de hipoglucemia hiperinsulinémica tumoral basadas en las cohortes retrospectivas de Anlauf y colaboradores (19), Rothmund y colaboradores (20), Nikfarjam y colaboradores (21), Zhao y colaboradores (22), Crippa y colaboradores (23) y Akca y colaboradores (24).

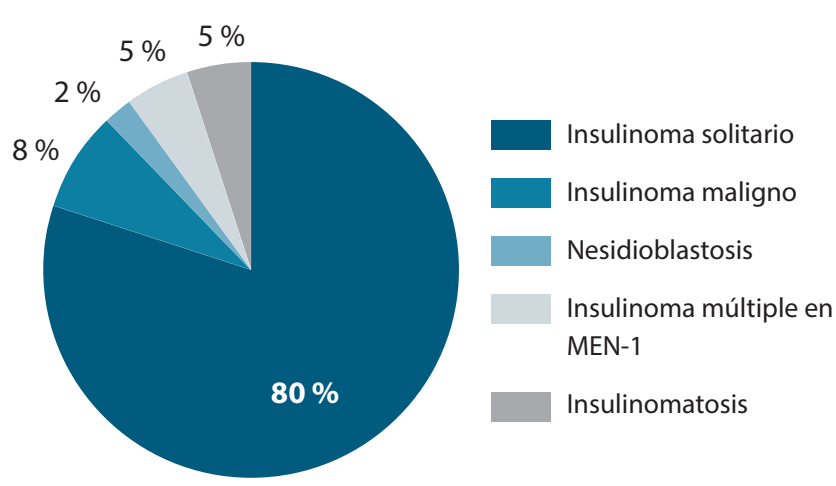

Los tumores neuroendocrinos pancreáticos (TNEP) son raros; $2-10 \%$ de tumores pancreáticos son neuroendocrinos y a su vez aproximadamente $7 \%$ de todos los tumores neuroendocrinos (TNE) son pancreáticos (25) con una presentación usualmente en mujeres entre la sexta y séptima décadas de la vida (26). Se clasifican clínicamente en funcionales y no funcionales. Los primeros tienen síntomas por hipersecreción hormonal siendo los más comunes los insulinomas, gastrinomas, vipomas, glucagonomas y PPomas. Por otro lado, los TNEP no funcionales se asocian con progresión tumoral, y agregan una mayor morbilidad y mortalidad (27).

Los insulinomas son TNE que secretan insulina, por lo que causan hipoglucemia y son los TNEP más frecuentes (45\% de casos) (28-31). Alrededor del $10 \%$ son múltiples, $10 \%$ malignos y $16 \%$ están asociados con neoplasias endocrinas múltiple tipo 1 (MEN-1). Los insulinomas que son múltiples o recurren, pero no están asociados a MEN-1 son denominados insulinomatosis (19).

El objetivo de esta revisión es ilustrar sobre la insulinomatosis, exponiendo los casos reportados, sus bases genéticas, el diagnóstico y el tratamiento.

\section{Obtención de los datos}

Se revisaron reportes y series de casos en PubMed, Medline y Google Scholar hasta el 30 de julio 2020, utilizando los términos insulinomatosis, multicentric insulinoma, multiple insu- 
linomas, refractory insulinoma, hereditary insulinoma y family insulinoma para identificar insulinomas múltiples. Se incluyeron los artículos con posibles casos de insulinomatosis que cumplieran con la definición: múltiples insulinomas MEN-1 negativo o recurrencia de insulinoma siendo MEN-1 negativo. En caso de que no se encontrara el artículo completo, se utilizó la información del resumen debido a que la cantidad de publicaciones sobre el tema es limitada. Se excluyeron los casos que habían sido reescritos y se consideró únicamente el reporte con mayores datos. Además, se descartaron casos que no tuvieran clara la evaluación de otras neoplasias o heredabilidad y que no cumplieran con la definición de insulinomatosis.

\section{El espectro de la hipoglucemia hiperinsulinémica tumoral}

El insulinoma es el TNE más frecuente, con una incidencia de 3-10 casos por millón/año (32). El $90 \%$ de los casos son unifocales y esporádicos (sin síndrome heredofamiliar) y el $10 \%$ de casos corresponde a un síndrome heredofamiliar como la neoplasia endocrina múltiple tipo 1 (MEN-1) o la neurofibromatosis tipo 1 (NF-1) (Figura 1) (19).

El MEN-1 se caracteriza por la presencia de hiperparatiroidismo primario, adenomas hipofisarios anteriores, TNEP y otras lesiones. Los TNEP más frecuentes en MEN-1 son gastrinomas e insulinomas. El insulinoma afecta al $10 \%$ de los pacientes con MEN-1 (33). A diferencia de los insulinomas esporádicos, que típicamente se presentan como lesiones solitarias, benignas y encapsuladas, los insulinomas en MEN-1 se desarrollan más temprano, tienden a ser multifocales, pueden comprometer todo el páncreas $(34,35)$ y se pueden asociar con otros TNEP funcionales (36) y con nesidioblastosis (37). Otras patologías neoplásicas poco frecuentes que producen hipoglucemia hiperinsulinémica endógena son la nesidioblastosis no asociada con MEN-1 y la insulinomatosis (Figura 1). Los insulinomas múltiples que no están asociados con MEN-1/NF-1/Von Hippel-Lindau (VHL) se denominan insulinomatosis (19).

\section{Historia de la insulinomatosis}

Desde 1906 se han descrito tumores múltiples en islotes pancreáticos (38), pero solo hasta 1977 se describió el primer caso de insulinomas múltiples en una mujer de 28 años que consultó por hipoglucemia hiperinsulinémica en una familia con DM, cuestionando la patogénesis de los insulinomas y su asociación con DM. Se le realizó una pancreatectomía parcial con resección de un insulinoma de 0,5 x 0,5 cm; pocos meses después, la hipoglucemia hiperinsulinémica recurrió, resecándosele 3/4 partes del páncreas 2 años más tarde; la hipoglucemia persistió y se le prescribió diazóxido. El padre de esta paciente requirió enucleación de un insulinoma a los 38 años y, luego, una pancreatectomía parcial a los 51 años por recidiva; a los 58 años recurrió la hipoglucemia hiperinsulinémica y se prescribió diazóxido (39).
En 2009, Anlauf utilizó el término de insulinomatosis para describir y comparar 14 casos (4,98\%) de insulinomas múltiples en 281 sujetos con hipoglucemia hiperinsulinémica recolectados entre 1975 y 2006 sin cirugía bariátrica previa, diferenciando los insulinomas solitarios, los múltiples heredofamiliares y los no familiares en diferentes centros europeos; fueron microtumores o macrotumores múltiples que expresaban exclusivamente insulina, sin mutaciones MEN-1/NF1. 4/14 casos fueron hombres, 13/14 casos fueron benignos con baja actividad proliferativa, 6/14 casos tuvieron hipoglucemia recurrente (con una mediana de recurrencia de 8,4 años) y un caso tenía historia familiar de insulinomatosis (19).

En 2018, Iacovazzo y colaboradores caracterizaron genéticamente 2 familias caucásicas asociando la mutación MafA en p.Ser64Fe con el síndrome insulinomatosis/DM autosómico dominante. En la primera familia con 29 sujetos evaluados, 7 resultaron con insulinomatosis y 6/7 fueron mujeres; hubo 13 casos con hiperglucemia/DM, de los cuales 9/13 fueron hombres y 4 tenían cataratas o glaucoma congénito. En la segunda familia, reportada en 1977, con 7 sujetos evaluados, 3 tenían insulinomatosis y 2/3 eran mujeres; 4 hombres tenían DM, no hay presencia de cataratas o glaucoma. Adicionalmente, reportaron 9 casos de insulinomatosis esporádica, 1/9 fue un hombre (dos casos previamente reportados). La edad promedio al diagnóstico fue de 39,4 \pm 13,1 años, el tamaño de los tumores fue de 0,4-1,1 cm y ninguno fue maligno (16).

Hasta la fecha, 108 casos cumplen criterios para insulinomatosis, con un rango de presentación entre 17 y 68 años, con mayor incidencia entre la cuarta y quinta décadas de la vida; 1 de cada 5 casos son hombres, alrededor del $3 \%$ son malignos y se han reportado en todas las razas (Tablas 1 y 2$)(15,16$, 19-24, 39-51).

\section{Comprendiendo la oncogénesis de la insulinomatosis}

Las células $\beta$ diferenciadas tienen la capacidad de secretar insulina en respuesta a la exposición a la glucosa, y los TNEP productores de insulina se caracterizan por el incremento en la masa y función de las células $\beta$. No se comprenden bien los mecanismos que regulan y controlan el crecimiento de las células $\beta$, pero algunos factores fisiológicos y múltiples genes locales y sistémicos están involucrados en su promoción y crecimiento (52-62).

Algunos casos de insulinomatosis se han asociado con alteraciones de MafA, un factor de transcripción con un diseño $\beta$-zip perteneciente a la familia de factores de transcripción MafA, que incluyen a MAfB, MAf y NRL. La proteína MafA se une al elemento promotor de la insulina RIPE3b, del gen promotor de la insulina, a $340 \mathrm{pb}$ corriente arriba del inicio, activando la expresión del gen de la insulina (63-66). La cooperación sinérgica de MafA con NeuroD y Pdx1 aumenta la síntesis y secreción de insulina. Además, MafA coordina con MafB la 


\begin{tabular}{|c|c|c|c|c|c|c|c|c|c|c|c|}
\hline Autor & Año & $\begin{array}{l}\text { Número de } \\
\text { pacientes }\end{array}$ & Sexo & $\begin{array}{l}\text { Edad } \\
\text { (años) }\end{array}$ & $\begin{array}{c}\text { Síntomas de } \\
\text { hipoglucemia }\end{array}$ & $\begin{array}{l}\text { Antecedente fami- } \\
\text { liar de diabetes/ } \\
\text { insulinoma }\end{array}$ & $\begin{array}{l}\text { Diagnóstico } \\
\text { inicial }\end{array}$ & MEN-1 & $\begin{array}{l}\text { Tratamiento } \\
\text { inicial }\end{array}$ & Recurrencia & Tratamiento final \\
\hline \multirow[t]{2}{*}{$\begin{array}{l}\text { Tragl y Mayr } \\
\text { (39) }\end{array}$} & 1977 & 2 & M & 28 & $\begin{array}{l}\text { Alteración en el } \\
\text { estado de cons- } \\
\text { ciencia y sudor }\end{array}$ & Sí & $\begin{array}{c}1 \text { insulinoma }(0,5 \\
\mathrm{cm} \times 0,5 \mathrm{~cm})\end{array}$ & Negativo & $\begin{array}{c}\text { Pancreatectomía } \\
\text { parcial }\end{array}$ & Sí & $\begin{array}{l}\text { Pancreatectomía y pos } \\
\text { teriormente diazóxido }\end{array}$ \\
\hline & & & $\mathrm{H}$ & 38 & $\begin{array}{l}\text { Síntomas de } \\
\text { hipoglucemia no } \\
\text { especificados }\end{array}$ & Sí & $\begin{array}{l}1 \text { pequeño insu- } \\
\text { linoma }\end{array}$ & Negativo & $\begin{array}{l}\text { Pancreatectomía } \\
\text { parcial }\end{array}$ & Sí & Diazóxido y dieta \\
\hline $\begin{array}{l}\text { Rosman y } \\
\text { colaborado- } \\
\text { res (41) }\end{array}$ & 2007 & 1 & $\mathrm{H}$ & 34 & $\begin{array}{l}\text { Letargo, } \\
\text { desmayo y des- } \\
\text { orientación }\end{array}$ & No & Nesidioblastosis & Negativo & Cirugía & $\begin{array}{l}\text { Sí (insulinoma } \\
\text { metastásico a } \\
\text { hígado) }\end{array}$ & $\begin{array}{l}\text { Ablación paliativa con } \\
\text { radiofrecuencia de las } \\
\text { lesiones hepáticas y } \\
\text { egreso con dexameta- } \\
\text { sona y verapamilo }\end{array}$ \\
\hline $\begin{array}{l}\text { Sheikh y } \\
\text { colaborado- } \\
\text { res (42) }\end{array}$ & 2007 & 1 & $\mathrm{H}$ & 32 & ND & No & $\begin{array}{c}1 \text { nódulo de } 1 \mathrm{x} \\
1,5 \mathrm{~cm}\end{array}$ & Negativo & Cirugía & Sí & Cirugía \\
\hline $\begin{array}{l}\text { Ishigaki y } \\
\text { colaborado- } \\
\text { res (43) }\end{array}$ & 2009 & 1 & M & 34 & $\begin{array}{l}\text { Pérdida de cons- } \\
\text { ciencia }\end{array}$ & No & 6 insulinomas & $\begin{array}{l}\text { No clínica } \\
\text { MEN-1 }\end{array}$ & Cirugía & ND & ND \\
\hline $\begin{array}{l}\text { Menassa- } \\
\text { Moussa (44) }\end{array}$ & 2010 & 1 & M & 33 & Convulsiones & No & $\begin{array}{c}1 \text { insulinoma de } \\
1 \mathrm{~cm}\end{array}$ & Negativo & Cirugía & Sí & $\begin{array}{l}\text { Diazóxido y octreotida } \\
\text { por comorbilidades del } \\
\text { paciente }\end{array}$ \\
\hline $\begin{array}{l}\text { Chen y co- } \\
\text { laboradores } \\
\text { (45) }\end{array}$ & 2011 & 1 & M & 47 & $\begin{array}{c}\text { Debilidad genera- } \\
\text { lizada y sensación } \\
\text { de hambre }\end{array}$ & No & $\begin{array}{l}\text { Múltiples tumo- } \\
\text { res pancreáticos } \\
\text { micro y macro. El } \\
\text { más grande de } \\
1,7 \mathrm{~cm}\end{array}$ & Negativo & Cirugía & Sí & $\begin{array}{l}\text { Pancreatectomía sub- } \\
\text { total }(75 \%)\end{array}$ \\
\hline $\begin{array}{l}\text { Guoqing y } \\
\text { colaborado- } \\
\text { res (46) }\end{array}$ & 2012 & 1 & M & 43 & Confusión y fatiga & No & $\begin{array}{l}2 \text { insulinomas } \\
(0,2 \text { y } 0,4 \mathrm{~cm})\end{array}$ & Negativo & Cirugía & Sí & $\begin{array}{l}2 \text { reintervenciones } \\
\text { quirúrgicas }\end{array}$ \\
\hline $\begin{array}{l}\text { Babic y co- } \\
\text { laboradores } \\
\text { (47) }\end{array}$ & 2016 & 1 & M & 66 & $\begin{array}{l}\text { Confusión, mareo, } \\
\text { alteración del es- } \\
\text { tado de conscien- } \\
\text { cia y del habla }\end{array}$ & No & $\begin{array}{l}\text { Múltiples insu- } \\
\text { linomas. El más } \\
\text { grande de } 4 \mathrm{~mm}\end{array}$ & Negativo & Cirugía & No & ND \\
\hline $\begin{array}{l}\text { Snaith y } \\
\text { Chipps (48) }\end{array}$ & 2017 & 1 & M & 40 & ND & No & $\begin{array}{l}\text { Insulinoma } \\
\text { benigno }\end{array}$ & Negativo & Cirugía & Sí & $\begin{array}{l}\text { Pancreatectomía total } \\
\text { + diazóxido y everoli- } \\
\text { mus (no tolerados) }\end{array}$ \\
\hline
\end{tabular}


Tabla 1. Series de casos o reportes de casos con posible diagnóstico de insulinomatosis (continuación)

\begin{tabular}{|c|c|c|c|c|c|c|c|c|c|c|c|}
\hline Autor & Año & $\begin{array}{l}\text { Número de } \\
\text { pacientes }\end{array}$ & Sexo & $\begin{array}{l}\text { Edad } \\
\text { (años) }\end{array}$ & $\begin{array}{c}\text { Síntomas de } \\
\text { hipoglucemia }\end{array}$ & $\begin{array}{l}\text { Antecedente fami- } \\
\text { liar de diabetes/ } \\
\text { insulinoma }\end{array}$ & $\begin{array}{l}\text { Diagnóstico } \\
\text { inicial }\end{array}$ & MEN-1 & $\begin{array}{l}\text { Tratamiento } \\
\text { inicial }\end{array}$ & Recurrencia & Tratamiento final \\
\hline \multirow[t]{11}{*}{$\begin{array}{l}\text { lacovazzo y } \\
\text { colaborado- } \\
\text { res (16) }\end{array}$} & 2018 & $\begin{array}{c}19 \\
(4 \text { casos } \\
\text { reportados } \\
\text { previamente })\end{array}$ & $\mathrm{H}$ & 25 & ND & $\begin{array}{l}\text { Sí (mutación MafA } \\
\text { identificada) }\end{array}$ & pT1m N0 M0 & Negativo & Cirugía & Sí & $\begin{array}{l}\text { Enucleación, } \\
\text { diazóxido + verapa- } \\
\text { milo }\end{array}$ \\
\hline & & & M & ND & ND & $\begin{array}{l}\text { Sí (mutación MafA } \\
\text { identificada) }\end{array}$ & $\begin{array}{l}\text { Insulinoma (no } \\
\text { hay especificacio- } \\
\text { nes disponibles) }\end{array}$ & Negativo & ND & ND & ND \\
\hline & & & M & 48 & ND & $\begin{array}{l}\text { Sí (mutación MafA } \\
\text { identificada) }\end{array}$ & $\begin{array}{l}\text { Insulinoma (no } \\
\text { hay especificacio- } \\
\text { nes disponibles) }\end{array}$ & Negativo & $\begin{array}{c}\text { octreotida + } \\
\text { verapamilo + } \\
\text { dexametasona }\end{array}$ & ND & ND \\
\hline & & & M & 44 & ND & $\begin{array}{l}\text { Sí (mutación MafA } \\
\text { identificada) }\end{array}$ & pT1m NO MO & Negativo & Cirugía & Sí & Octreotida + diazóxido \\
\hline & & & M & 53 & ND & $\begin{array}{l}\text { Sí (mutación MafA } \\
\text { identificada) }\end{array}$ & $\begin{array}{l}\text { Insulinoma (no } \\
\text { hay especificacio- } \\
\text { nes disponibles) }\end{array}$ & Negativo & Verapamilo & ND & ND \\
\hline & & & M & 46 & ND & $\begin{array}{l}\text { Sí (mutación MafA } \\
\text { identificada) }\end{array}$ & pT1m NO MO & Negativo & Cirugía & Sí & $\begin{array}{l}\text { Pancreatectomía par- } \\
\text { cial, luego pancreatec- } \\
\text { tomía total }\end{array}$ \\
\hline & & & M & 18 & ND & $\begin{array}{l}\text { Sí (mutación MafA } \\
\text { identificada) }\end{array}$ & $\begin{array}{l}\text { Insulinoma (no } \\
\text { hay especificacio- } \\
\text { nes disponibles) }\end{array}$ & Negativo & $\begin{array}{c}\text { Cirugía + octreo- } \\
\text { tida }\end{array}$ & Sí & $\begin{array}{l}\text { verapamilo + dexame- } \\
\text { tasona }\end{array}$ \\
\hline & & & M & 38 & ND & $\begin{array}{l}\text { Sí (mutación MafA } \\
\text { identificada) }\end{array}$ & $\begin{array}{l}\text { Insulinoma (no } \\
\text { hay especificacio- } \\
\text { nes disponibles) }\end{array}$ & Negativo & Cirugía & Sí & $\begin{array}{l}\text { Pancreatectomía par- } \\
\text { cial + diazóxido }\end{array}$ \\
\hline & & & M & 28 & ND & $\begin{array}{l}\text { Sí (mutación MafA } \\
\text { identificada) }\end{array}$ & $\begin{array}{l}\text { Insulinoma (no } \\
\text { hay especificacio- } \\
\text { nes disponibles) }\end{array}$ & Negativo & Cirugía & Sí & $\begin{array}{l}\text { Pancreatectomía } \\
\text { parcial x 2, diazóxido, } \\
\text { pancreatectomía total }\end{array}$ \\
\hline & & & M & 55 & ND & $\begin{array}{l}\text { Sí (mutación MafA } \\
\text { identificada) }\end{array}$ & $\begin{array}{l}\text { Insulinoma (no } \\
\text { hay especificacio- } \\
\text { nes disponibles) }\end{array}$ & Negativo & Diazóxido & ND & ND \\
\hline & & & M & 17 & ND & No & pT1m N0 M0 p & Negativo & Cirugía & Sí & $\begin{array}{l}\text { Enucleación, diazóxido } \\
\text { + dexametasona, } \\
\text { Whipple }\end{array}$ \\
\hline
\end{tabular}


Tabla 1. Series de casos o reportes de casos con posible diagnóstico de insulinomatosis (continuación)

\begin{tabular}{|c|c|c|c|c|c|c|c|c|c|c|c|}
\hline Autor & Año & $\begin{array}{l}\text { Número de } \\
\text { pacientes }\end{array}$ & Sexo & $\begin{array}{l}\text { Edad } \\
\text { (años) }\end{array}$ & $\begin{array}{c}\text { Síntomas de } \\
\text { hipoglucemia }\end{array}$ & $\begin{array}{l}\text { Antecedente fami- } \\
\text { liar de diabetes/ } \\
\text { insulinoma }\end{array}$ & $\begin{array}{l}\text { Diagnóstico } \\
\text { inicial }\end{array}$ & MEN-1 & $\begin{array}{l}\text { Tratamiento } \\
\text { inicial }\end{array}$ & Recurrencia & Tratamiento final \\
\hline & & & $M$ & 48 & ND & No & pT1m No Mo & Negativo & $\begin{array}{l}\text { Diazóxido + } \\
\text { prednisona }\end{array}$ & Sí & $\begin{array}{l}\text { Pancrectomía parcial, } \\
\text { diazóxido, octreotida }\end{array}$ \\
\hline & & & M & 64 & ND & No & pT1m NO MO & Negativo & Cirugía & Sí & $\begin{array}{l}\text { Pancreatectomía } \\
\text { parcial }\end{array}$ \\
\hline & & & M & 47 & ND & No & pT1m No Mo & Negativo & Cirugía & No & ND \\
\hline & & & M & 51 & ND & No & pT1m No Mo & Negativo & Cirugía & No & ND \\
\hline & & & $\mathrm{H}$ & 55 & ND & No & pT1m No Mo & Negativo & Cirugía & ND & ND \\
\hline & & & M & 28 & ND & No & pT1m No Mo & Negativo & Cirugía & No & ND \\
\hline & & & $M$ & 37 & ND & No & pT1m No Mo & Negativo & Cirugía & ND & ND \\
\hline & & & M & 20 & ND & No & pT1m NO MO & Negativo & Cirugía & ND & ND \\
\hline $\begin{array}{l}\text { Akca y cola- } \\
\text { boradores } \\
\text { (24) }\end{array}$ & 2018 & 15 & $\begin{array}{c}2 \mathrm{H} \\
13 \mathrm{M}\end{array}$ & $46^{* *}$ & ND & ND & $\begin{array}{l}\text { Múltiples micro y } \\
\text { macroadenomas }\end{array}$ & ND & Cirugía & Sí (6/15) & ND \\
\hline $\begin{array}{l}\text { Akiko y co- } \\
\text { laboradores } \\
\text { (49) }\end{array}$ & 2018 & 2 & ND & ND & ND & ND & ND & ND & ND & ND & ND \\
\hline $\begin{array}{l}\text { Shigeru y } \\
\text { colaborado- } \\
\text { res (50) }\end{array}$ & 2018 & 2 & ND & ND & ND & ND & ND & ND & ND & ND & ND \\
\hline $\begin{array}{l}\text { Alencary } \\
\text { colaborado- } \\
\text { res (51) }\end{array}$ & 2019 & 1 & M & 52 & ND & No & $\begin{array}{l}\text { Múltiples insuli- } \\
\text { nomas }\end{array}$ & ND & Cirugía & Sí & Pancreatectomía distal \\
\hline $\begin{array}{l}\text { González- } \\
\text { Devia y } \\
\text { colaborado- } \\
\text { res (15) }\end{array}$ & 2019 & 1 & M & 57 & $\begin{array}{l}\text { Síntomas neuro- } \\
\text { glucopénicos }\end{array}$ & Sí & $\begin{array}{l}\text { Múltiples insuli- } \\
\text { nomas }\end{array}$ & Negativo & Cirugía & Sí & $\begin{array}{l}\text { Pancreatectomía total } \\
\text { y diazóxido }\end{array}$ \\
\hline
\end{tabular}




\begin{tabular}{|c|c|c|c|c|c|c|c|c|c|c|c|c|}
\hline Autor & $\begin{array}{l}\text { Número de } \\
\text { pacientes }\end{array}$ & $\begin{array}{c}\text { Edad } \\
\text { promedio }\end{array}$ & Sexo & $\begin{array}{l}\text { Número de } \\
\text { pacientes } \\
\text { MEN-1 } \\
\text { positivo }\end{array}$ & $\begin{array}{c}\text { Número de } \\
\text { pacientes con } \\
\text { nesidoblastosis }\end{array}$ & $\begin{array}{l}\text { Número de } \\
\text { pacientes } \\
\text { con insulino- } \\
\text { ma maligno }\end{array}$ & $\begin{array}{l}\text { Número de } \\
\text { pacientes } \\
\text { con insulino- } \\
\text { ma solitario }\end{array}$ & $\begin{array}{l}\text { Número de } \\
\text { pacientes } \\
\text { con NF-1 }\end{array}$ & $\begin{array}{l}\text { Número de } \\
\text { pacientes con } \\
\text { múltiples insuli- } \\
\text { nomas }\end{array}$ & $\begin{array}{l}\text { Número de } \\
\text { pacientes con } \\
\text { recurrencias de } \\
\text { insulinomas }\end{array}$ & $\begin{array}{c}\text { Posibles } \\
\text { casos de insu- } \\
\text { linomatosis }\end{array}$ & $\begin{array}{c}\text { Antecedentes } \\
\text { familiares } \\
\text { de diabetes/ } \\
\text { insulinoma }\end{array}$ \\
\hline $\begin{array}{l}\text { Rothmund } \\
\text { y colabora- } \\
\text { dores (20) }\end{array}$ & 396 & ND & ND & 13 & 30 & 84 & 240 & 0 & $\begin{array}{c}-29 \text { MEN-1 } \\
\text { positivos } \\
-13 \text { MEN-1 } \\
\text { negativos }\end{array}$ & $27(6,4 \%)$ & 13 & ND \\
\hline $\begin{array}{l}\text { Hellman y } \\
\text { colaborado- } \\
\text { res }(40)\end{array}$ & 65 & 48,4 & $\begin{array}{l}45 \mathrm{H} \\
20 \mathrm{M}\end{array}$ & 6 & 3 & 7 & $\begin{array}{l}47 \text { ( } 2 \text { casos } \\
\text { MEN-1 } \\
\text { positivos) }\end{array}$ & 0 & $\begin{array}{l}-2 \text { MEN-1 } \\
\text { positivos } \\
-6 \text { MEN-1 } \\
\text { negativos }\end{array}$ & $\begin{array}{l}2 \text { casos con in- } \\
\text { sulinoma previo } \\
\text { y } 2 \text { reinterven- } \\
\text { ciones }\end{array}$ & 6 & ND \\
\hline $\begin{array}{l}\text { Nikfarjam y } \\
\text { colaborado- } \\
\text { res (21) }\end{array}$ & 61 & 53 & $\begin{array}{l}26 \mathrm{H} \\
35 \mathrm{M}\end{array}$ & 3 & 0 & 5 & $\begin{array}{l}56 \text { (4 MEN-1 } \\
\text { positivos) }\end{array}$ & 0 & $\begin{array}{l}-3 \mathrm{MEN}-1 \\
\text { positivos } \\
-2 \mathrm{MEN}-1 \\
\text { negativos }\end{array}$ & $\begin{array}{l}\text { - } 1 \text { MEN positivo } \\
\text { - } 1 \text { MEN negativo }\end{array}$ & 3 & ND \\
\hline $\begin{array}{l}\text { Anlauf y } \\
\text { colabora- } \\
\text { dores/ } 2009 \\
\text { (19) }\end{array}$ & 281 & ND & ND & 14 & 0 & 0 & 253 & 1 & $\begin{array}{l}-13 \text { MEN } 1 \\
\text { positivo } \\
-14 \text { MEN } 1 \\
\text { negativos }\end{array}$ & \begin{tabular}{|l}
-4 de los 13 \\
MEN1 positivo \\
-6 de los 14 MEN \\
1 negativos
\end{tabular} & 14 & $\begin{array}{c}\text { Un caso } \\
\text { (Familiar con } \\
\text { adenomatosis } \\
\text { familiar de } \\
\text { células Beta) }\end{array}$ \\
\hline $\begin{array}{l}\text { Zhao y co- } \\
\text { laboradores } \\
\text { (22) }\end{array}$ & 292 & $\begin{array}{c}41,7 \pm \\
14,6\end{array}$ & $\begin{array}{l}123 \mathrm{H} \\
169 \mathrm{M}\end{array}$ & 17 & 0 & 13 & 247 & 0 & $\begin{array}{c}-17 \text { MEN-1 } \\
\text { positivos } \\
-15 \text { MEN-1 } \\
\text { negativos }\end{array}$ & NA & 15 & ND \\
\hline $\begin{array}{l}\text { Crippa y } \\
\text { colaborado- } \\
\text { res (23) }\end{array}$ & 198 & 48 & $\begin{array}{c}82 \mathrm{H} \\
116 \mathrm{M}\end{array}$ & 11 & 0 & 7 & 129 & 0 & $\begin{array}{l}-9 \text { MEN } \\
\text { positivos } \\
-7 \text { MEN } \\
\text { negativos }\end{array}$ & $\begin{array}{l}-2 \text { MEN } \\
\text { positivos } \\
-4 \text { MEN } \\
\text { negativos }\end{array}$ & 11 & ND \\
\hline
\end{tabular}

$\mathrm{ND}=$ no disponible; $\mathrm{M}=$ mujer; $\mathrm{H}=$ hombre

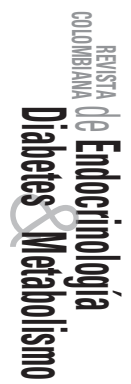


generación y diferenciación de las células $\beta$ (67). MafA regula el balance de glucosa y energía en diferentes tejidos como el adiposo, del páncreas y muscular; se asocia con la secreción de insulina estimulada por glucosa, sirviendo como un barómetro de la secreción de insulina; y su deficiencia conduce a DM y nefropatía diabética en ratones (68), por lo que desempeña un papel esencial en la producción y mantenimiento de las células $\beta$ maduras (69-71).

La sobreexpresión de MafA estimula la expresión de Pdx1, MafA, Nkx2.2, Nkx6.1, Ngn3, Isl1 e insulina (72). Las mutaciones halladas en MafA en insulinomatosis o DM son a nivel de p.Ser64Fe, lo que genera una fosforilación en el dominio N-terminal transactivador; esto conlleva un aumento de la actividad MafA endógena, que incrementa la estabilidad y actividad en las células $\beta$ (16).

Los niveles más altos de proteína y de actividad incrementada del p.Ser64Fe mutado inducen a la expresión de genes involucrados en la regulación del ciclo celular, como el CCND2, un blanco de MafA y regulador clave de la proliferación de las células $\beta$, que causa la transformación y aparición de insulinomatosis. La mutación p.Ser64Fe altera la regulación estricta de la estabilidad de MafA en respuesta a los cambios en la concentración de glucosa; esto afecta la secreción de insulina estimulada por la glucosa y explica la generación de DM. Sin embargo, no se conocen completamente los mecanismos que permiten que la misma mutación genética conduzca a DM o insulinomatosis (16).

Algunos pacientes desarrollan primero DM2 y luego insulinomatosis. No obstante, muchas veces las 2 patologías son mutuamente excluyentes, y los factores individuales determinan si se presenta una patología u otra. Por su parte, se ha visto que la DM2 se desarrolla más en hombres, e insulinomatosis más en mujeres (16).

\section{Criterios diagnósticos para insulinomatosis}

Se definió el síndrome insulinomatosis/DM como el trastorno autosómico dominante caracterizado por hipoglucemia hiperinsulinémica, insulinomas multicéntricos, DM2 o intolerancia a la glucosa; algunos pacientes pueden presentar historia personal o familiar de cataratas o glaucoma congénito $(16$, 73). Se requiere la documentación de la hipoglucemia hiperinsulinémica, la presencia sincrónica de micro o macrotumores de células $\beta$ o la recurrencia de la hipoglucemia hiperinsulinémica con una lesión nueva de células $\beta$ en caso de historia de un insulinoma previo. También se debe evaluar la presencia personal o familiar de hiperglucemia/DM2, cataratas/glaucoma congénito $(15,16,19-24,39-51)$.

A nivel histológico (Figura 2), la tinción de inmunohistoquímica es positiva para insulina, cromogranina A y sinaptofisina; debe ser negativa para glucagón (66) y otras hormonas. Se deben excluir: neoplasias endocrinas familiares (MEN-1, neoplasia endocrina múltiple tipo 4 [MEN-4], VHL,
NF-1, esclerosis tuberosa, entre otras), otros síndromes heredofamiliares $y$ otras neoplasias neuroendocrinas extrapancreáticas; y debe documentarse la secuenciación del gen MEN-1 negativa y secuenciar el gen MafA para definir la heredabilidad. Por otra parte, es indispensable hacer seguimiento a los pacientes a quienes se les diagnostica insulinomas solitarios esporádicos, ya que alrededor del $5 \%$ de insulinomas solitarios esporádicos pueden presentar una nueva hipoglucemia hiperinsulinémica en el transcurso de semanas a 2 décadas y se podría diagnosticar insulinomatosis (Figura 1) $(15,16,19-24,39-51)$.

Figura 2. Histología de la insulinomatosis. Tinción de hematoxilina y eosina, $10 \mathrm{x}$, apreciándose microtumores y macrotumores bien diferenciados en el páncreas (15).

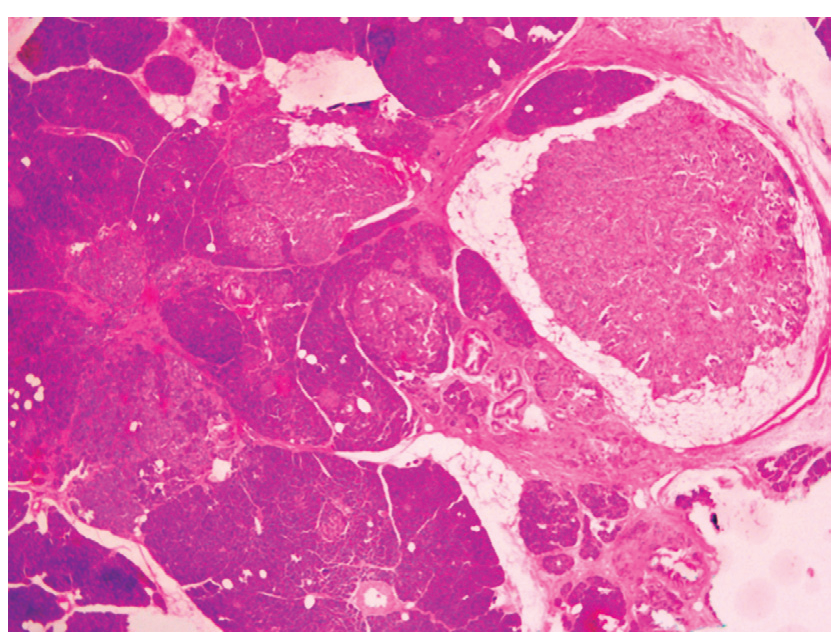

\section{Manejo y pronóstico de la insulinomatosis}

La base terapéutica de la insulinomatosis es la cirugía con intención de controlar los síntomas de la hipoglucemia hiperinsulinémica. A la fecha, no se puede considerar la curación por la naturaleza recurrente de la condición y un potencial maligno bajo. La cirugía puede ser enucleación, pancreatectomía parcial, pancreatectomía subtotal o pancreatectomía total con seguimiento periódico tanto de los síntomas como de imágenes. En casos irresecables, se puede considerar el manejo local o sistémico. A nivel local, se encuentra la radiofrecuencia del lecho pancreático o de las metástasis hepáticas; a nivel sistémico, se encuentran el diazóxido, los corticoides, los análogos de somatostatina, el verapamilo y el everolimus $(15,16,19-24,39-51)$.

\section{Conclusión}

La insulinomatosis es una enfermedad muy rara caracterizada por la presencia de insulinomas múltiples sincrónicos o metacrónicos, sin otros TNEP; con una alta tasa de recurrencia en el páncreas residual, que puede requerir pancreatectomía total; ocasionalmente producen metástasis; no se asocia con 
síndromes heredofamiliares clásicos como MEN-1, VHL, MEN4, NF1 o esclerosis tuberosa; no hay un antecedente de cirugía bariátrica; puede ser heredofamiliar o esporádica; y es más frecuente en mujeres adultas. La mutación MafA puede ser un conductor. El seguimiento a largo plazo de los insulinomas solitarios esporádicos puede detectar más casos de insulinomatosis. Se recomienda la evaluación genética y el seguimiento a largo plazo a los insulinomas múltiples o recidivantes.

\section{Fuente de financiamiento}

No aplicable.

Todos los autores confirman que han trabajado en la atención de pacientes con tumores neuroendocrinos de páncreas, que participaron en la concepción o diseño del trabajo; o la adquisición, análisis o interpretación de datos para el trabajo y la redacción del trabajo o su revisión crítica para el contenido intelectual importante. Todos participaron en la aprobación final de la versión que se publicó y están de acuerdo con ser responsables de todos los aspectos del trabajo para garantizar que las preguntas relacionadas con la precisión o la integridad de cualquier parte del trabajo se investiguen y resuelvan adecuadamente.

\section{Agradecimientos}

Al Dr. Carlos Olimpo Mendivil.

\section{Conflictos de interés}

Todos los autores declaran que no tienen ningún conflicto de interés en esta publicación.

\section{Referencias}

1. Banting FG, Best $\mathrm{CH}$. The internal secretion of pancreas. J Lab Clin Med.1922;7(5):251-66.

2. Whipple AO, Frautz VK. Adenomas of islet cells with hyperinsulinism: a review. Ann Surg. 1935;101(6):1299-335.

3. Harris S. Hyperinsulinism and dysinsulinism. JAMA. 1924;83(10):729733.

4. Parker JT, Finley CS. Some results obtained by the treatment of nervous phenomena with glucose. Proc Soc Exper Biol Med. 1924;21(8):517-9.

5. Wolf A, Hare CC, and Riggs HW. Neurological Manifestations in Two Patients with Spontaneous Hypoglycemia; with Necropsy Report of a Case of Pancreatic Island Adenomata. Bull Neurol Inst. 1933;3:232.

6. Harris S. Hyperinsulinism, a Definite Disease Entity. JAMA. 1933;101(25):1958-65.

7. Harris S. Epilepsy and Narcolepsy Associated with Hyperinsulinism. JAMA. 1933;100(5):321-328.

8. Harris S. The Diagnosis of Surgical Hyperinsulinism. Southern Surgeon. 1934;3:199.

9. Harris S Nomenclature of the Disorders of Insulin Secretion; Diabetes Mellitus, Hyperinsulinism and Dysinsulinism. Ann Int Med.1934:7:1084-100.

10. Wilder RM. Hyperinsulinism (Colver Lecture). Internat Clin. 1933;2:1-18.

11. Wauchope GM. Critical Review. Hypoglycemia. Qt J Med. 1933;2:117.

12. Restrepo K, Garavito G, Rojas L, et al. Nesidioblastosis del adulto coexistente con insulinoma: Reporte de caso. Rev Colomb Cancerol. 2009;13(1):49-60.

13. Leong AS, Slavotinek AH, Higgins BA. Nesidioblastosis, islet cell hyperplasia, and adenomatosis in a case of metastasizing insulinoma: contribution to the genesis of the islets of Langerhans. Diabetes Care. 1980;3(4):53742.

14. Bettini V, Martini C, Ferrata M, et al. Hypoglycemic Syndrome Recurrence after Surgical Removal of a Pancreatic Neuroendocrine Adenoma. Abstract \#958. $11^{\text {th }}$ Annual ENETS Conference; 2014

15. González-Devia D, Gómez-Escobar G, López-Panqueva R, et al. Recurrent Hypoglycemia Related By Sporadic Malignant Insulinomatosis: Case Report. Abstract \# 009, 16th Annual ENETS Conference 2019 [Internet]. Enets.org. 2020 [citado el 14 de agosto de 2020]. Disponible en: https:// www.enets.org/16th_annual_enets_conference.html

16. Iacovazzo D, Flanagan SE, Walker E, et al. MAFA missense mutation causes familial insulinomatosis and diabetes mellitus. Proceedings of the National Academy of Sciences. 2018;115(5):1027-32.

17. Schmitt J, Boullu-Sanchis S, Moreau F, et al. Association of malignant insulinoma and type 2 diabetes mellitus: a case report. Ann Endocrinol. 2008;69(1):69-72.

18. Sakurai A, Aizawa T, Katakura M, et al. Insulinoma in a patient with Non-Insulin-dependent Diabetes Mellitus. Endocrine Journal. 1997,44(4):473-7.

19. Anlauf M, Bauersfeld J, Raffel A, Koch CA, Henopp T, Alkatout I, et al. Insulinomatosis: multicentric insulinoma disease that frequently causes early recurrent hyperinsulinemic hypoglycemia. Am J Surg Pathol. 2009;33(3):339-46.
20. Rothmund M, Angelini L, Brunt LM, et al. Surgery for benign insulinoma: an international review. World J Surg. 1990;14(3):393-8.

21. Nikfarjam M, Warshaw AL, Axelrod L, et al. Improved contemporary surgical management of insulinomas: a 25-year experience at the Massachusetts General Hospital. Ann Surg. 2008;247(1):165-72.

22. Zhao YP, Zhan HX, Cong L, Zhang TP, Liao Q Dai MH, et al. Risk factors for postoperative pancreatic fistula in patients with insulinomas: analysis of 292 consecutive cases. Hepatobiliary Pancreat Dis Int 2012;11(1):102 106.

23. Crippa S, Zerbi A, Boninsegna L, Capitanio V, Partelli S, Balzano G, et al Surgical management of insulinomas: short- and long-term outcomes after enucleations and pancreatic resections. Arch Surg. 2012;147(3):261-6.

24. Akca A, Wirowski D, Starke AAR, Goretzki PE. Follow-Up in Adult Patients with Pancreatogenic Hypoglycemia Caused by Sporadic Micro- or MacroInsulinomatosis: 16 Years of Experience in One Center. J Diabetes Treat. 2018;JDBT-144.

25. Lawrence B, Gustafsson BI, Chan A, et al. The epidemiology of gastroen teropancreatic neuroendocrine tumors. Endocrinol Metab Clin North Am. 2011;40(1):1-18, vii.

26. Mckenna LR, Edil BH. Update on pancreatic neuroendocrine tumors 2014;3(4):258-75

27. Guzmán YF, López RP, Vera A, et al. Herramientas para el abordaje diagnóstico de los tumores neuroendocrinos de páncreas. Rev Colomb Cir 2018,33(1):79-99.

28. Vanderveen K, Grant C. Insulinoma. Cancer Treat Res. 2010;153:235-52.

29. Guettier JM, Gorden P. Insulin secretion and insulin-producing tumors. Expert Rev Endocrinol Metab. 2010;5(2):217-27

30. De Herder WW, Niederle B, Scoazec JY, et al. Well-differentiated pancreatic tumor/carcinoma: insulinoma. Neuroendocrinology. 2006;84(3):183-8.

31. Vinik A, Perry RR, Casellini C, Hughes MS, Feliberti E. Pathophysiology and treatment of pancreatic neuroendocrine tumors (PNETs): new developments. InEndotext [Internet] 2018 Jun 12. MDText. com, Inc.

32. Dadan J, Wojskowicz P, Wojskowicz A. Neuroendocrine tumors of the pancreas. Wiad Lek. 2008;61(1-3):43-7

33. Rich TA, Perrier ND. Multiple endocrine neoplasia syndromes. Surg Clin North Am. 2008;88(4):863-95.

34. Demeure MJ, Klonoff DC, Karam JH, et al. Insulinomas associated with multiple endocrine neoplasia type I: the need for a different surgical approach. Surgery. 1991;110:998-1005.

35. O'Riordain DS, Brien T, van Heerden JA, et al. Surgical management of insulinoma associated with multiple endocrine neoplasia type I. World J Surg. 1994;18:488-94

36. Wills B, López R, González-Devia, et al. Papel de la ultrasonografía intraoperatoria en sujetos con tumores neuroendocrinos de páncreas con sospecha de NEM 1. Rev. Colomb. Radiol. 2014;25(4):4090-5.

37. Le Bodic MF, Heymann MF, Lecomte M, et al. Immunohistochemical study of 100 pancreatic tumors in 28 patients with multiple endocrine neoplasia, type I. Am J Surg Pathol. 1996;20(11):1378-84. 
38. Herxheimer, G. Ueber Pankreascirrhose (bei Diabetes). Arch f path Anat $\mathrm{u}$ Phys. 1906:183:228.

39. Tragl KH, Mayr WR. Familial islet-cell adenomatosis. Lancet. 1977;2:426-

40. Hellman P, Goretzki P, Simon D, Dotzenrath C, Röher HD. Therapeutic experience of 65 cases with organic hyperinsulinism. Langenbecks Arch Surg. 2000;385(5):329-36

41. Rosman J, Bravo-Vera R, Sheikh A, Gouller A. Metastatic insulinoma in an adult patient with underlying nesidioblastosis. J Endocrinol Invest. 2007;30(6):521-4.

42. Sheikh A, Zuberi L, Haque N. Rare among the rarities--recurrent insulinoma. J Coll Physicians Surg Pak. 2007;17(6):364-6.

43. Ishigaki S, Yoshihara A, Ohwada R, Hizuka N, Hatori T, Furukawa T, et al. Insulinoma with six islet cell tumors associated with severe hypoglycemia. Intern Med. 2009;48(12):1041-6.

44. Menassa-Moussa L, Halaby G, Braidy C. Multiple pancreatic insulinomas: multislice CT. Abdom Imaging. 2010;35(6):690-3.

45. Chen PY, Wu TJ, Ou HY, et al. Applying intraoperative insulin level monitoring for tumor removal in a patient with recurrent pancreatic multiple insulinomas. J Formos Med Assoc. 2011;110(6):410-4.

46. Guoqing Y, Dou-Jingtao D, Li Z, Yiming MA. Special Form of Pancreatic Hyperinsulinemic Hypoglycemia -Insulinomatosis: A Case Report. Endocrine Reviews. 2014;35(3): SUN-0288.

47. Babic B, Keutgen X, Nockel P, Miettinen M, Millo C, Herscovitch P, et al. Insulinoma Due to Multiple Pancreatic Microadenoma Localized by Multimodal Imaging. I Clin Endocrinol Metab. 2016;101(10):3559-63.

48. Snaith J y Chipps D. Will this ever end? Recurrent hypoglycaemia due to insulinomatosis. Poster Presentation \#271. The Joint Annual Scientific Meetings of the Endocrine Society of Australia and the Society for Reproductive Biology 2017. Clin Endocrinol. 2018;89:86.

49. Akiko K, Tomoko N, Nobuyuki O, Ja-Mun C. Insulinomatosis: A case repart [Internet]. Iglobal.jst.go.jp. 2020 [citado el 20 de agosto de 2020]. Disponible en: https://jglobal.jst.go.jp/en/detail?JGLOBAL ID $=201802250401216649$

50. Kawabata S, Minamiguchi S, Kataoka TR, Umakoshi H, Haga H, Moriyoshi K. Insulinomatosis, Two cases of pancreatic insulinomatosis [Internet]. Sasappa.co.jp. 2020 [citadado el 20 de agosto de 2020]. Disponible en: http://www.sasappa.co.jp/online/abstract/jjdp/1/035/ html/0910350207.html

51. Alencar N, Nunes M, Segatelli V, Castanheira T, Seraphim C, Pereira M. Insulinomatosis: A Rare Cause of Recurrent Hyperinsulinemic Hypoglycemia. Presentation Number SUN-320 Journal of the Endocrine Society. 2019;3(suppl 1):SU-320.

52. Parsons JA, Brelje TC, Sorenson RL. Adaptation of islets of Langerhans to pregnancy: increased islet cell proliferation and insulin secretion correlates with the onset of placental lactogen secretion. Endocrinology. 1992;130:1459-66.

53. Rieck S, White P, Schug J, et al. he transcriptional response of the islet to pregnancy in mice. Mol Endocrinol. 2009;23:1702-12.

54. Alonso LC, Yokoe T, Zhang P, et al. Glucose infusion in mice: a new model to induce beta-cell replication. Diabetes. 2007;56(7):1792-801.

55. Cano DA, Rulifson IC, Heiser PW, et al. Regulated $\beta$-cell regeneration in the adult mouse pancreas. Diabetes. 2008;57(4):958-66.
56. Brüning JC, Winnay J, Bonner-Weir S, et al. Development of a novel polygenic model of NIDDM in mice heterozygous for IR and IRS-1 null alleles. Cell. 1997;88(4):561-72.

57. Terauchi, Y Takamoto I, Kubota N, et al Glucokinase and IRS-2 are required for compensatory beta cell hyperplasia in response to high-fat diet-induced insulin resistance. J Clin Invest. 2007;117:246-57.

58. Porat S, Weinberg-Corem N, Tornovsky-Babaey S, et al. Control of pancreatic $\beta$ cell regeneration by glucose metabolism. Cell metabolism. 2011;13(4):440-9.

59. Bernard C, Thibault C, Berthault MF, et al. Pancreatic beta-cell regeneration after 48-h glucose infusion in mildly diabetic rats is not correlated with functional improvement. Diabetes. 1998;47(7):1058-65.

60. Drucker DJ. The biology of incretin hormones. Cell metabolism 2006;3(3):153-65.

61. Flier SN, Kulkarni RN, Kahn CR. Evidence for a circulating islet cell growth factor in insulin-resistant states. Proceedings of the National Academy of Sciences. 2001;98(13):7475-80.

62. Yuchi Y, Cai Y, Legein B, De Groef S, Leuckx G, Coppens V, et al. Estrogen Receptor $\alpha$ Regulates $\beta$-Cell Formation During Pancreas Development and Following Injury. Diabetes. 2015;64(9):3218-28.

63. Diana ZY, Tai MH, Linning KD, et al. MafA expression and insulin promoter activity are induced by nicotinamide and related compounds in INS-1 pancreatic beta-cells. Diabetes. 2006;55(3):742-50.

64. Ye DZ, Tai MH, Linning KD, et al. MafA expression and insulin promoter activity are induced by nicotinamide and related compounds in INS-1 pancreatic beta-cells. Diabetes. 2006;55(3):742-50.

65. Matsuoka TA, Kaneto H, Miyatsuka T, et al. Regulation of MafA expres sion in pancreatic betacells in $\mathrm{db} / \mathrm{db}$ mice with diabetes. Diabetes. 2010;59(7):1709-20.

66. Zhang C, Moriguchi T, Kajihara M, et al. MafA is a key regulator of glucosestimulated insulin secretion. Mol Cell Biol. 2005;25(12):4969-76.

67. Kaneto H, Matsuoka TA, Katakami N, Matsuhisa M. Combination of MafA, PDX-1 and NeuroD is a useful tool to efficiently induce insulin-producing surrogate beta-cells. Curr Med Chem. 2009;16(24):3144-51

68. Tsuchiya $\mathrm{M}$, Tsuchiya $\mathrm{K}$, Yasuda $\mathrm{K}$, et al. MafA is a key molecule in glucose and energy balance in the central nervous system and peripheral organs. Int J Biomed Sci. 2011;7(1):19-26.

69. Nishimura W, Takahashi S, Yasuda K. MafA is critical for maintenance of the mature beta cell phenotype in mice. Diabetologia. 2015;58(3):566-74.

70. Tsuchiya M, Misaka R, Nitta K, Tsuchiya K. Transcriptional factors, Mafs and their biological roles. World J Diabetes. 2015;6(1):175-83.

71. Chiou SH, Chen SJ, Chang YL, et al. MafA promotes the reprogramming of placenta-derived multipotent stem cells into pancreatic islets-like and in sulin+ cells. J Cel Mol Med. 2011;15(3):612-24.

72. Dayer D, Tabandeh MR, Moghimipour E, et al. MafA Overexpression: A New Efficient Protocol for In Vitro Differentiation of Adipose-Derived Mesenchymal Stem Cells into Functional Insulin-Producing Cells. Cell J. 2019;21(2):169-78.

73. Islet cell adenomatosis (Concept Id: C1578917) - MedGen - NCBI [Internet]. Ncbi.nlm.nih.gov. 2020 [citado el 20 de agosto de 2020]. Disponible: https://www.ncbi.nlm.nih.gov/medgen/C1578917 


\section{Jardiance}

(empagliflozina)

\section{Trayenta}

(linagliptina) $5 \mathrm{mg}$ comprimidos
Trayenta'Duo

(linagliptina/metformina HCl)

\section{Simplicidad reforzada.}

(Q. Reducción de HbA1c ${ }^{1}$

if Reducción de presión

Eficaciaa

arterial sistólica ${ }^{1}$

(i) Reducción del peso

y masa corporal'

Bajas tasas de hipoglicemia ${ }^{2}$

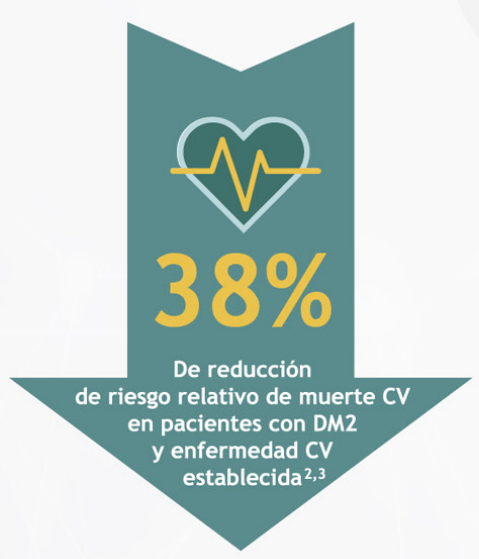

Jardiance Duo

(empagliflozina/metformina $\mathrm{HCl}$ )
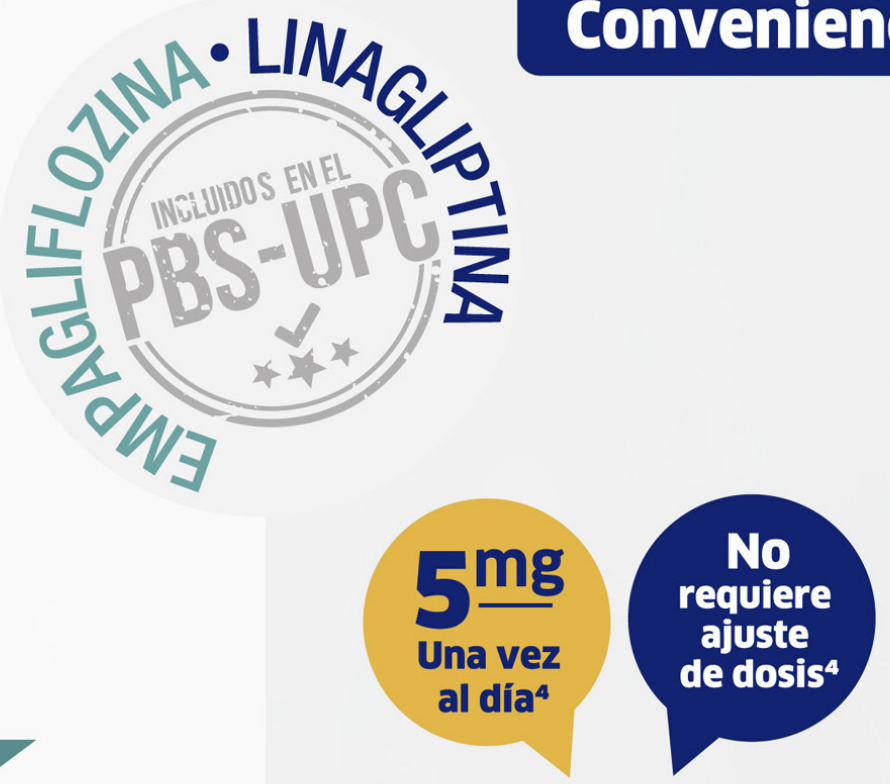

Seguridad CV y renal, sin incrementar el riesgo de hospitalización por IC 5,6

\section{Conveniencia"}

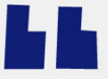

TRATAMIENTO PARA EL MES COMPLETO.

Glyxambi (empagliflozina/ linagliptina)

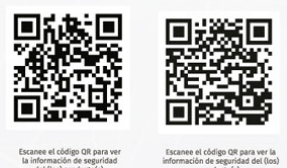

